\title{
NOTE ON AFFINELY CONNECTED MANIFOLDS
}

\author{
SHIING-SHEN CHERN
}

The aim of this note is to prove some statements concerning the differential geometry in the large of an affinely connected manifold.

Let $M$ be an orientable differentiable manifold of dimension $n$ and class two. We say that an affine connection is defined in $M$, if a set of quantities ${ }^{1} \Gamma_{i j}^{k}$ is defined in each allowable coordinate system $x^{i}$ such that under change of the allowable coordinate system they are transformed according to the following law:

$$
\bar{\Gamma}_{p q}^{r}=\frac{\partial^{2} x^{k}}{\partial \bar{x}^{p} \partial \bar{x}^{q}} \frac{\partial \bar{x}^{r}}{\partial x^{k}}+\Gamma_{i j}^{k} \frac{\partial x^{i}}{\partial \bar{x}^{p}} \frac{\partial x^{j}}{\partial \bar{x}^{q}} \frac{\partial \bar{x}^{r}}{\partial x^{k}} .
$$

The connection may be symmetric or asymmetric.

It is well known that from $\Gamma_{i j}^{k}$ the covariant derivative of a contravariant vector $X^{i}$ can be defined as follows:

$$
X_{, k}^{i}=\frac{\partial X^{i}}{\partial x^{k}}+X^{l} \Gamma_{l k}^{i} .
$$

We also recall that the affine curvature tensor is given by

$$
R_{j k l}^{i}=\frac{\partial \Gamma_{j l}^{i}}{\partial x^{k}}-\frac{\partial \Gamma_{j k}^{i}}{\partial x^{l}}-\Gamma_{m l}^{i} \Gamma_{j k}^{m}+\Gamma_{m l s}^{i} \Gamma_{j l}^{m}
$$

We put

$$
R_{k l}=R_{i k l}^{i}
$$

and introduce the exterior differential form

$$
P=R_{k l} d x^{k} d x^{l} .
$$

Then the main theorem of this note can be stated as follows:

TheOREM. The integral of $P$ over any two-dimensional cycle is equal to zero.

To prove this theorem we consider $n$ linearly independent contravariant vectors $X_{(1)}^{i}, \cdots, X_{(n)}^{i}$ and their determinant

Received by the editors February 8, 1947.

${ }^{1}$ All indices in this paper run from 1 to $n$ and we agree as usual that repeated indices mean summation. 


$$
\Delta=\epsilon_{i_{1}} \cdots i_{n} X_{(1)}^{i_{1}} \cdots X_{(n)}^{i_{n}},
$$

where $\epsilon_{i_{1}} \ldots i_{n}$ is the Kronecker index. When the vectors are parallelly transported, we have

$$
-\frac{d \Delta}{\Delta}=\Gamma_{i k}^{i} d x^{k} .
$$

The differential form $-d \Delta / \Delta$ we shall denote for simplicity by $\phi$. It now follows immediately from (3) that its exterior derivative is

$$
d \phi=2 P .
$$

It is sufficient for the proof of this theorem to assume that the domain of integration is a simplicial two-dimensional cycle in a sufficiently fine simplicial decomposition of $M$ such that each simplex lies in one coordinate neighborhood of $M$, as every cycle over which the integral of $P$ is defined can be approximated by a simplicial cycle with the above property. On such a simplicial cycle we shall define a continuous field of $n$ independent contravariant vectors at each point. According to a standard procedure in the theory of fibre bundles, ${ }^{2}$ the sets of vectors are at first defined at the vertices of the cycle. They can be extended over the one-dimensional simplexes, because $M$ is orientable. To extend the field over the two-dimensional simplexes and hence over the whole cycle, we notice that the sets of $n$ independent contravariant vectors at a point whose determinant is positive (or negative) form a topological space which is the group space of the group of linear transformations in $n$ variables with positive (or negative) determinant and is hence simply-connected. ${ }^{3}$ As the sets of vectors defined on the boundary of a simplex give rise to a mapping of the boundary into the group space in question, the simply-connectedness of this group space implies the possibility of extension over the two-dimensional simplexes. The integral of $P$ over the cycle can now be interpreted as the integral of $P$ over this field of independent contravariant vectors in the space of all sets of $n$ independent contravariant vectors of the manifold $M$. From (8) it follows that the integral is zero and the theorem is proved.

With the terminology of the cohomology theory the theorem can be stated as follows: The cohomology class to which $P$ belongs is the zero class.

We shall state the following corollaries of our theorem:

${ }^{2}$ Cf., for instance, N. Steenrod, Topological methods for the construction of tensor functions, Ann. of Math. vol. 43 (1942) pp. 116-131.

${ }^{3}$ A proof is given later in this paper. 
COROLlaRy 1. Let $M$ be a compact orientable affinely-connected manifold of class two and even dimension $n$. Then

$$
\int_{M} P^{n / 2}=0
$$

or

$$
\int_{M} \epsilon^{i_{1}} \cdots i_{n} R_{i_{1} i_{2}} R_{i_{8} i_{4}} \cdots R_{i_{n-1} i_{n}} d x^{1} \cdots d x^{n}=0 .
$$

The integrand of the integral (9a):

$$
I=\epsilon^{i_{1}} \cdots i_{n} R_{i_{1} i_{2}} R_{i_{8} i_{4}} \cdots R_{i_{n-1} i_{n}}
$$

is a tensor density of the affine connection.

Corollary 2. Let $M$ have the same meaning as in Corollary 1. Then I can not be always positive nor always negative in $M$.

Remark. In terms of the notation of Elie Cartan, the affine connection can be written as the infinitesimal displacement of affine frames $p e_{1} \cdots e_{n}$ :

$$
d p=\omega^{i} \mathfrak{e}_{i}, \quad d \mathfrak{e}_{i}=\omega_{i}^{j} \hat{e}_{j},
$$

with the equations of structure:

$$
d \omega^{i}=\omega^{i} \omega_{j}^{i}+\Omega^{i}, \quad d \omega_{j}^{i}=\omega_{j}^{k} \omega_{k}^{i}+\Omega_{j}^{i} .
$$

Then we have

$$
P=\Omega_{i}^{i} \text {. }
$$

I shall conjecture that all the differential forms

$$
P_{\alpha}=\Omega_{i_{1}}^{i_{2}} \Omega_{i_{2}}^{i_{3}} \cdots \Omega_{i_{\alpha}}^{i_{1}}, \quad 0 \leqq \alpha \leqq n / 4,
$$

define cohomology classes which are topological invariants of $M$. I hope to return to this in a later paper.

For completeness I indicate here a proof of the well known fact that the space of all matrices (with a natural topology) $\left(a_{i j}\right),\left|a_{i j}\right|>0$, is simply connected, as I am not able to give the exact reference. This space is a group manifold and the theorem in question follows from the following theorem which I quote from Chevalley in a slightly different version: ${ }^{4}$

4. Chevalley, Theory of Lie groups, p. 59. 
Let $G$ be a connected Lie group and $H$ a subgroup of $G$. If the homogeneous space $G / H$ is simply connected, the fundamental group of $G$ is isomorphic to a factor group of the fundamental group of $H$.

Using this theorem we shall give the proof by induction on the order $n$ of the matrices. Let $G$ be the group of all these matrices, $H$ the subgroup of all matrices

$$
\left(\begin{array}{cccc}
1 & a_{12} & \cdots & a_{1 n} \\
0 & a_{22} & \cdots & a_{2 n} \\
\cdot & \cdot & \cdot & \cdot \\
0 & a_{n 2} & \cdots & a_{n n}
\end{array}\right)
$$

and $K$ the subgroup of all matrices

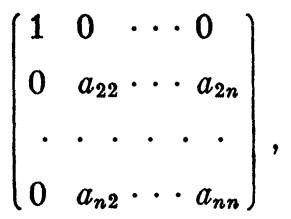

so that

$$
K \subset H \subset G \text {. }
$$

The induction hypothesis states that $K$ is simply connected. It is easy to verify that both homogeneous spaces $H / K$ (the space of right cosets) and $G / H$ (the space of left cosets) are Euclidean spaces and hence are simply connected. It therefore follows from the above theorem that $H$ and hence also $G$ are simply connected.

Institute of Mathematics, Academia Sinica 